INVESTIGACIONES

\title{
Creencias docentes de profesores ejemplares y su incidencia en las prácticas pedagógicas*
}

\author{
Teacher's beliefs of proficient teachers and their impact on pedagogical practices \\ Crenças do mestre de professores e proficients ther impacto \\ sobre práticas pedagógicas
}

\section{Karina Cortez Quevedo, Valeria Fuentes Quelin, Isabel Villablanca Ortiz, Carolina Guzmán ${ }^{\mathrm{a}}$}

\author{
${ }^{a}$ Universidad de Valparaíso, Facultad de Medicina, Escuela de Psicología. Valparaíso, Chile. Telf.: (32) \\ 2508611. Correo electrónico: carolina.guzmán@uv.cl
}

\begin{abstract}
RESUMEN
El principal propósito de esta investigación fue analizar las creencias docentes que están a la base de las prácticas pedagógicas de 3 profesores ejemplares noveles insertos en el Colegio San Antonio de la comuna de Villa Alemana. Inscrita en una aproximación cualitativa, la información fue recabada por medio de 2 entrevistas realizadas a cada uno de los docentes y 8 observaciones no participantes dentro del aula. A partir de los datos obtenidos, se pudo comprender de manera acabada los procesos que se llevan a cabo en el contexto de enseñanza-aprendizaje. Los resultados permitieron conocer las principales creencias presentes en los docentes, sus similitudes y diferencias, así como la presencia de concordancia entre sus discursos y sus prácticas pedagógicas.
\end{abstract}

Palabras clave: creencias docentes, prácticas pedagógicas, profesores ejemplares, profesores noveles.

\begin{abstract}
The main purpose of this investigation was to analyze teachers' beliefs in the teaching practice's basis of 3 novel proficient teachers in San Antonio School located in Villa Alemana. Under a qualitative approach, the information was gathered through a set of 2 interviews done to each of the teachers and 8 passive observations in the classroom. From here it was possible to fully understand the processes that take place in the teaching-learning context. The obtained results allowed us to learn about the main beliefs that the novel proficient teachers had their similarities, and differences, as well as the identifiable agreement between their speech and their pedagogical practices.
\end{abstract}

Key words: : teachers' beliefs, pedagogical practice, proficient teachers, novel teachers.

\section{RESUMO}

O principal objetivo desta investigação foi analisar as crenças docentes que estão na base das práticas pedagógicas de 3 novatos e eficientes professores do Colégio de San Antonio, na comunidade de Villa Alemana. Em uma abordagem qualitativa, as informações foram recolhidas por meio de 2 entrevistas feitas a cada um dos professores e de 8 observações ouvintes em sala de aula. A partir dos dados obtidos, pode-se compreender os mecanismos imbricados no contexto do processo ensino-aprendizagem. Resultados permitiram conhecer as principais crenças que têm os professores, suas semelhanças e diferenças, bem como a presença de concordância entre seu discurso e suas práticas pedagógicas.

Palavras chave: crenças docentes, práticas pedagógicas, professores eficientes, professores novatos.

* Investigación de pregrado financiada por proyecto DIUV 30/2009 a cargo de la Dra. Carolina Guzmán V., Universidad de Valparaíso, Chile. 


\section{INTRODUCCIÓN}

Durante la década de los años ochenta, comienza a surgir un mayor interés por estudiar e indagar sobre la práctica, formación y desarrollo de los docentes, ${ }^{1}$ adquiriendo gran relevancia temas como la autopercepción del profesorado y la influencia de las historias de vida en las prácticas pedagógicas. Para tener una comprensión más acabada acerca de las prácticas pedagógicas y los procesos de enseñanza-aprendizaje, resulta necesario examinar tanto lo que ocurre dentro del aula, como lo que se encuentra a la base del actuar docente. Díaz et al. (2010) sostienen que, indagando el entramado de creencias que sostiene la praxis pedagógica que utiliza el profesor, es posible comprender su actuación y la concepción que tiene de la educación.

Evidenciar, por tanto, la incidencia que tienen las creencias docentes al momento de educar, permite dilucidar y justificar transformaciones tanto en las prácticas que se dan dentro del aula como en la formación inicial. Al indagar este ámbito, altamente relevante para la investigación educativa, se privilegia la formación íntegra y reflexiva, de modo que los docentes puedan (desde que inician su trabajo profesional) dar cuenta -de manera consciente- de las propias creencias y utilizarlas como guía de sus prácticas pedagógicas.

A partir de ese marco inicial, surgieron diversas preguntas directrices para sugerir tanto una búsqueda en la literatura actual como un diseño de investigación para avanzar en la respuesta a las siguientes interrogantes: ¿Qué tipo de creencias docentes poseen los profesores que muestran mejores desempeños? ¿Las creencias docentes reconocidas por éstos, se plasman en sus prácticas pedagógicas?

El objetivo de la presente investigación fue analizar las creencias docentes que están a la base de las prácticas pedagógicas de tres profesores ejemplares insertos en un colegio particular subvencionado de la Región de Valparaíso (Chile). Lo anterior permitió conocer las principales creencias docentes de dichos profesores con respecto a procesos de enseñanza - aprendizaje, a sí mismos y a sus estudiantes; lo que luego nos permitió analizar la concordancia entre sus creencias y prácticas pedagógicas.

\section{FUNDAMENTACIÓN TEÓRICA}

\subsection{CREENCIAS DOCENTES}

Dentro de todo proceso de enseñanza-aprendizaje se ponen en juego una serie de variables que interactúan de manera constante y compleja, resultando así un proceso dinámico y difícil de analizar. El concepto de creencia forma parte de este proceso y "[...] se entiende como una parte de la dimensión personal, afectiva y emocional, íntimamente ligada a la propia cultura que se manifiesta en el ambiente en el que estamos y en el que configuramos, influyendo, a su vez, en nosotros y en lo que hacemos" (Oliver, 2009: 63), dando cuenta de un proceso que influirá en cada una de las decisiones que se tome, así como también en las prácticas que lleven a cabo. Se desprende de lo anterior que las creencias pedagógicas hacen alusión principalmente a concepciones que tienen los

1 Los términos "docente" y "profesor" serán utilizados para referirse tanto a los como a las educadoras, sin hacer distinción de género. 
docentes acerca de los diferentes procesos de enseñanza-aprendizaje, en su totalidad. Así las creencias pedagógicas “... (Son] personales, reconstruidas sobre la base de conocimientos pedagógicos históricamente elaborados y transmitidos a través de la formación y en la práctica pedagógica. Por lo tanto, son una síntesis de conocimientos culturales y de experiencias personales" (Cit. en De Vincenzi, 2009: 90).

Desde este punto de vista los docentes tienen bajo su responsabilidad un rol protagónico, no sólo como ejecutores de los procesos de enseñanza-aprendizaje, sino también, como principales motores del cambio en la educación, existiendo la necesidad de que los profesores sean capaces de promover cambios tanto en sus prácticas pedagógicas como en las concepciones que tienen acerca de la educación, de sus estudiantes y de las metodologías de aprendizaje (Díaz et al., 2010).

\subsection{PRÁCTICAS PEDAGÓGICAS}

Se entiende por práctica pedagógica “[...] la praxis social, objetiva e intencional en la que intervienen los significados, las percepciones y acciones de los agentes implicados en el proceso educativo" (Fierro et al., 1999: 20-21). Desde este punto de vista, las prácticas pedagógicas implican un marco de acción constituido por “[...] los procesos de formación académica, la experiencia profesional, las creencias, las concepciones pedagógicas, las políticas curriculares, la visión de mundo, la forma de ser de quien organiza y conduzca el trabajo en aula" (Cit. en Castro, 2007: 113). Estas prácticas están fundamentadas en aprendizajes adquiridos durante toda la formación educacional recibida por el profesional docente desde su niñez, esto en complemento a la existencia de representaciones acerca de lo qué es ser un buen profesor. Los docentes tienden a contextualizar sus prácticas en relación a lo vivenciado por ellos mismos como estudiantes, lo cual se caracteriza por tener creencias y valoraciones personales a la base (Latorre, 2004). Los docentes no pueden aislarse de su contexto socio-educativo y las diversas realidades y complejidades a las que se verán enfrentados, les permitirán estar aprendiendo y reaprendiendo a enseñar.

\subsection{PROFESORES EJEMPLARES}

Por medio de la observación de las prácticas pedagógicas es posible identificar algunas variables que se asocian a la figura del "profesor ejemplar", que en general son docentes con mucha experiencia profesional (expertos). Como menciona Medina Moya (Cit. en Guzmán et al., 2009: 19) "Los modelos formales utilizados por los principiantes en forma de normas y protocolos técnicos, se transforman y adaptan a lo largo de la experiencia, a través de la confrontación analítica e integración inteligente del conocimiento y la técnica que posee el profesor y la realidad del aula".

Dentro de las características identificadas en los docentes, cabe mencionar: tienen un dominio del contenido, poseen habilidades pedagógicas que le permitan transmitir los contenidos con claridad, se comunican de manera eficiente (Ochoa y Domínguez, 2007), conservan cercanía afectiva con los estudiantes, expresan un gusto por enseñar y goza de capacidades para enfrentar exitosamente las dificultades pedagógicas (López, 2010). Además, el profesor ejemplar es comprensivo, posee un concepto positivo de sí mismo y de su trabajo que le permite construir un ambiente agradable y estimulante en el aula. Este clima de aula contribuiría tanto a lograr un mejor aprendizaje de los contenidos 
formales como al fortalecimiento del desarrollo físico, intelectual y afectivo de los estudiantes (Gutiérrez, 2008). Brophy y Good (Cit. en Roehrig et al., 2007: 3), caracterizan los profesores ejemplares como aquellos docentes que enfatizan la instrucción académica, poseen expectativas positivas de los estudiantes, optimizan el tiempo de las tareas a realizar, consideran las necesidades del grupo curso, son capaces de relacionar el currículo con cosas cotidianas y relevantes para los estudiantes, siendo claros y entusiastas en la transmisión de instrucciones.

Tal como señala Hopkins (Cit. en Segovia, 2003: 7), "son múltiples las capacidades e indicadores de un buen desempeño profesional docente [...], pero ninguna propuesta por sí sola ofrece una visión global ni tampoco la garantía de que se logren 'buenos aprendizajes"”. Actualmente, si bien no existe una caracterización única de lo que sería un profesor ejemplar, los autores anteriormente expuestos, entre otros, brindan ciertos lineamientos que pueden ayudar a reconocer cuándo el docente lleva a cabo su labor de manera efectiva, y por ende, "ejemplar". Así, en nuestro país contamos con el Marco para la Buena Enseñanza (Gobierno de Chile, 2008), que delinea 4 dominios que ayudan a definir a un profesor ejemplar: Preparación de la enseñanza (dominio A), creación de un ambiente propicio para el aprendizaje (dominio B), enseñanza para el aprendizaje de todos los estudiantes (dominio C) y las responsabilidades profesionales (dominio D).

\subsection{PROFESORES NOVELES}

"Son considerados profesores noveles, a todos aquellos que posean menos de 3 años de experiencia en su quehacer profesional, aunque otros lo prolongan hasta los 5 primeros años" (Imbernon, 1994: 58). Johnston y Ryan los identifican de la siguiente manera:

Los profesores en su primer año de docencia son extranjeros en un mundo extraño, un mundo que le es conocido y desconocido a la vez. Aunque hayan dedicado miles de horas en las escuelas viendo a profesores e implicados en los procesos escolares, los profesores principiantes no están familiarizados con la situación específica en la que empiezan a enseñar (Cit. en Marcelo, 2009: 63).

Algunos estudios muestran que la experiencia adquirida en el primer año de docencia produce cambios significativos en las actitudes de los profesores, como en el manejo de grupo y en el ámbito disciplinar, adquiriendo conductas más autoritarias y controladas. Otros consideran que el proceso más relevante es el de socialización que se produce con las comunidades educativas y que comienza antes incluso de la graduación (Cit. en Marcelo, 2009: 69).

Cabe mencionar que este apartado no estaba previsto abordarlo en el comienzo de la investigación, ya que no se pensaba trabajar con docentes noveles, pero ha sido un elemento emergente de relevancia cuando se inició el trabajo de campo pues se esperaba encontrar características de docentes ejemplares en docentes de más años de experiencia en la labor docente. Este componente finalmente resulta ser un hallazgo en la presente investigación, considerando que docentes de pocos años de labor docente pueden poseer elementos característicos de un profesor de mayor experticia. 


\section{METODOLOGÍA}

\subsection{TIPO DE ESTUDIO}

Para llevar a cabo la presente investigación se utilizó una metodología cualitativa. Según Denzin y Lincoln (1994, 2008), este tipo de investigación es multimetódica, naturalista e interpretativa; esto supuso: la inmersión en la vida cotidiana de la situación a estudiar, la valoración y descubrimiento de la perspectiva del sujeto sobre su propio mundo y la consideración de la investigación como un suceso interactivo. Se buscó hacer emerger una realidad construida colectivamente que sirva de marco de comprensión para el fenómeno de estudio de manera holística. La forma en que se pudo acceder a este conglomerado de significados y representaciones fue a través de sus propios actores.

\subsection{ESTRATEGIA DE INVESTIGACIÓN}

La estrategia utilizada consistió en un estudio de casos múltiple de carácter instrumental. Este tipo de estudio permitió la validación de los resultados a partir de instancias de triangulación y posibilitó extender los resultados empíricos hacia fenómenos de similares condiciones y niveles más generales de teoría (Vasilachis, 2006). Cabe mencionar que el caso, en este estudio, tiene un interés secundario, desempeña un papel de apoyo, facilitando el entendimiento de alguna problemática (Arzaluz, 2005: 121), como las creencias que están a la base de las prácticas pedagógicas de los docentes ejemplares.

\subsection{PROCEDIMIENTO}

La ejecución de la investigación se realizó en cuatro fases; (i) Contacto con la institución educacional; (ii) Aplicación de encuestas a estudiantes y docentes y selección de profesores ejemplares; (iii) Recogida de información, obtención de datos en terreno por medio de entrevistas y observaciones; (iv) Análisis de datos a través del método de comparaciones constantes, interpretaciones y conclusiones.

\subsection{PARTICIPANTES}

Los participantes de esta investigación fueron tres profesores considerados "ejemplares", insertos en un colegio particular subvencionado de la comuna de Villa Alemana. La selección de éstos fue intencionada y basada en criterios (Patton, 1990). Se establecieron tres criterios para seleccionar a los pedagogos, aplicados sucesivamente. El primer criterio de elección, teniendo como referencia una investigación Fonide (Guzmán et al. 2009) en el tema de docentes ejemplares, consistió en recoger la percepción de la comunidad a través de encuestas aplicadas a estudiantes y docentes, en la que se les pedía recomendar a un profesor considerado como ejemplar. Una vez obtenida la lista de estos docentes, sólo se seleccionaron aquellos de sexo masculino ${ }^{2}$ y luego aquellos pertenecientes a diferentes disciplinas. Por último, todos lo participantes resultaron ser docentes noveles (con menos de 3 años de experiencia), tal y como se muestra en la Tabla 1.

2 Para dejar abierta la posibilidad de hacer una comparación de género en estudios futuros dentro de esta misma línea de trabajo. 
Tabla 1. Descripción General de Participantes

\begin{tabular}{|c|c|c|}
\hline Profesor & Asignatura & Años de Experiencia \\
\hline Docente A (C.H) & Física & 1 \\
\hline Docente B (C.E) & Historia y Geografía & 3 \\
\hline Docente C (C.A) & Matemática & 1 \\
\hline
\end{tabular}

\subsection{TÉCNICAS O INSTRUMENTOS DE RECOLECCIÓN DE INFORMACIÓN}

La indagación sobre las creencias docentes de cada participante, fue realizada por medio de dos entrevistas semi-estructuradas. La pauta de la primera entrevista fue transversal a todos los participantes y la pauta de la segunda entrevista fue diferente para cada participante, se construyó de manera personalizada, según la información recaba en la primera entrevista y de acuerdo a lo observado en el aula. Además, se llevaron a cabo ocho observaciones no participantes, cada una de una hora pedagógica (en total 24 observaciones), lo que permitió obtener información sobre las prácticas docentes en el aula de cada participante. Éstas se llevaron a cabo de manera detallada y precisa, quedando plasmadas en notas de campo.

\subsection{CRITERIOS PARA ASEGURAR LA RIGUROSIDAD DE LA INVESTIGACIÓN}

Los métodos utilizados que rigieron esta investigación para asegurar su rigurosidad, fueron:

a) Transferibilidad: Recolección abundante de datos descriptivos.

b) Dependencia: Métodos solapados (entrevistas y las observaciones de campo).

c) Confirmabilidad: Triangulación de las diferentes perspectivas de las investigadoras.

\subsection{ANÁLISIS DE DATOS}

Para analizar las observaciones y entrevistas, se utilizó el método de las comparaciones constantes propuestos por Glaser y Strauss (Cit. en Osses et al., 2006: 119-133) a través del programa Atlas ti. En primera instancia se llevó a cabo la segmentación del corpus de datos, para posteriormente ser codificados y categorizados en unidades de significado relevantes para los propósitos de este estudio. Posteriormente se realizó la categorización. La primera fase de categorización se ejecutó a través de las categorías deductivas construidas a partir de la literatura y a medida que se realizó el proceso de categorización de las unidades de significado, surgieron nuevas categorías a partir de los datos, vale decir, inductivas, las cuales se fueron comparando y contrastando con las deductivas y entre sí mismas.

\section{RESULTADOS}

A partir del análisis del corpus de datos (entrevistas y observaciones) se obtuvo un total de 82 categorías (codes): 16 deductivas y 66 inductivas. Sin embargo, para los 
propósitos de este paper se ha realizado una selección de las categorías con el fin de presentar los principales resultados del estudio. En las Tablas 2 y 3 se dan a conocer los codes deductivos e inductivos seleccionados con sus respectivas frecuencias.

Tabla 2. Categorías Deductivas y sus frecuencias

\begin{tabular}{|c|c|}
\hline Nombre de la categoría & F \\
\hline Creencias con respecto a la vocación por la enseñanza & 5 \\
\hline Creencias acerca de la importancia de la cercanía afectiva y cognitiva & 37 \\
\hline Creencias en relación a la escucha activa del docente hacia el estudiante & 19 \\
\hline Creencias con respecto al estudiante y la adaptación de metodologías y \\
estrategias & 10 \\
\hline Creencias acerca de los métodos de manejo conductual y disciplinarios & 55 \\
\hline Creencias acerca del uso de herramientas prácticas & 15 \\
\hline Creencias sobre la utilización de ejemplos y aplicación para el aprendizaje & 23 \\
\hline Creencias acerca de las capacidades y expectativas de los estudiantes & 22 \\
\hline Creencias con respecto a la autonomía del estudiante en torno a su proceso \\
de enseñanza-aprendizaje & 19 \\
\hline
\end{tabular}

Tabla 3. Categorías Inductivas y sus frecuencias

\begin{tabular}{|c|c|}
\hline Nombre de la categoría & F \\
\hline Creencias sobre la autoridad docente & 7 \\
\hline Creencias con respecto a un profesor ejemplar & 4 \\
\hline Creencias con respecto a los hitos de la formación docente & 3 \\
\hline Creencias con respecto al docente novel y su formación & 12 \\
\hline Creencias sobre el docente como guía o mediador del contenido & 25 \\
\hline Creencias acerca del docente orientador & 5 \\
\hline Creencias con respecto a la estructuración de la clase & 3 \\
\hline Creencias con respecto a la valoración de conocimientos previos y & 11 \\
\hline diagnóstico & 6 \\
\hline Creencias con respecto al bienestar socio-emocional de los estudiantes & 16 \\
\hline Creencias sobre la formación de estudiantes en valores & 43 \\
\hline Creencias acerca de las estrategias motivacionales y participativas & 18 \\
\hline Creencias acerca de la apreciación personal y académica & 3 \\
\hline Creencias sobre el aprendizaje significativo & 7 \\
\hline Creencias con respecto a la creatividad & 2 \\
\hline
\end{tabular}




\begin{tabular}{|c|c|}
\hline Creencias acerca de los estilos de aprendizaje & 9 \\
\hline Creencias con respecto a las diferencias de género en cuanto a las habilidades & 5 \\
\hline Creencia sobre la participación activa & 10 \\
\hline $\begin{array}{c}\text { Creencias acerca de la etapa de desarrollo del estudiante y su } \\
\text { comportamiento }\end{array}$ & 4 \\
\hline Creencias con respecto a la motivación intrínseca y la extrínseca & 4 \\
\hline Creencias con respecto al esfuerzo vinculado al éxito & 2 \\
\hline
\end{tabular}

A continuación se presentan en detalle los hallazgos con respecto a las principales creencias y prácticas pedagógicas de los profesores ejemplares.

\subsection{CREENCIAS CON RESPECTO A LA VOCACIÓN DEL PROFESOR}

Los docentes concordaron en la creencia de que la vocación y la pasión son elementos fundamentales a la hora de impartir una asignatura, puesto que por medio de ellas se logran satisfacciones en el quehacer profesional (Física) y se consiguen mejores resultados al momento de motivar a los estudiantes (Historia), ya que para enseñar no basta sólo el interés por una disciplina (Matemática). Estas creencias concuerdan con lo mencionado por Hansen (Cit. en Day, 2006: 32-33), quien considera que una persona que tenga un sentido de vocación, desempeña su labor más plenamente que alguien que sólo lo considere un trabajo.

\subsection{CREENCIAS ACERCA DEL ROL Y CARACTERÍSTICAS}

DOCENTE EN TORNO A LA AUTORIDAD Y EL PERFIL DE UN PROFESOR EJEMPLAR

En relación a la autoridad, el profesor de Física mostró en la práctica su manejo del grupo con estrategias poco invasivas y sin acudir a los gritos. El guardar silencio para él es suficiente para dar a entender a los estudiantes su posición.

(Docente A) "los estudiantes gritan en el aula, él deja de escribir y guarda silencio, en ese momento entre los mismos estudiantes comienzan a pedir silencio."

Por el contrario, el profesor de Matemática mencionó sentir que la autoridad es un ámbito que aún no maneja y que cree que en ocasiones "se le va de las manos", porque no existen límites en la relación profesor - estudiante. De esta manera, la principal diferencia entre ambos docentes radica en el equilibrio que logran tener dentro del aula entre la cercanía y distanciamiento con los estudiantes. Para el profesor de Historia la autoridad tiene intima relación con el liderazgo, donde éste último sería el que permite un buen manejo de grupo. A lo anterior el profesor de Matemática añade que un profesor ejemplar es aquel que a lo largo de su trayectoria ha ido trabajando en pro de mejoras de su quehacer y posee un adecuado manejo de los contenidos y la didáctica a la hora de explicar a sus estudiantes; tal y como él lo experimentó cuando era estudiante.

\subsection{CREENCIAS CON RESPECTO A LA FORMACIÓN Y PROGRESO DEL DOCENTE}

En este apartado cobra relevancia el hecho de que los docentes participantes de esta investigación cuentan con menos de 3 años de experiencia, por lo tanto, poseen ciertas 
características y creencias amparadas en sus pocos años de labor profesional. Los tres docentes concordaron en que el primer año de experiencia es el más importante dentro de la carrera docente. Los docentes manifestaron creer que es en este año donde se siente o no el gusto por enseñar (Física), se enfrentan por primera vez a un grupo de niños o adolescentes y deben poner las reglas de su labor para con ellos (Historia) y que sólo la experiencia les da los insumos necesarios para enfrentar nuevas problemáticas (Matemática).

Otro aspecto relevante de mencionar relacionado con los noveles y vinculado con el comienzo en la labor docente, es la inseguridad o ansiedad que éstos evidenciaron sobre todo durante los primeros tres años (Cit. en Díaz y Núñez, 2008). Los docentes creen de manera similar que la poca experiencia lleva a los profesores ser menos asertivos en sus prácticas. Sin embargo, creen de manera certera que por medio de la práctica esto es solucionable al adquirir mayor conocimiento. Desde este punto de vista se situaron también en concordancia con respecto a seguir perfeccionando sus habilidades, conocimientos y estrategias pedagógicas, lo cual conllevaría a mejorar su labor, repercutiendo de manera positiva en el desempeño de los estudiantes.

(Docente B) "yo, siempre me estoy capacitando, leyendo por mi cuenta siempre sabiendo un poco más, cosa de que el niño no se vaya simplemente con lo que se le está pidiendo para la prueba, si no que él sepa algo más."

\subsection{CREENCIAS EN RELACIÓN AL DOCENTE COMO}

FACILITADOR EN EL APRENDIZAJE DE CONTENIDOS

En lo que respecta al rol, los tres profesores coincidieron en que el docente debe mediar el contenido durante los procesos de enseñanza-aprendizaje, guiando al estudiante, para que finalmente éste dé con la respuesta correcta. Tal como señala Doyle (Cit. en Cruz, 2008: 144) "[...] la enseñanza se concibe como un proceso que facilita el aprendizaje [...]”; donde el docente es el guía y otorga al estudiante un papel activo en el proceso de crecimiento y cambio que es el aprendizaje.

(A) "el alumno sabe todo pero no lo ha descubierto por así decirlo, entonces uno pasa a ser solamente un mediador nada más, entonces esa idea es la que siempre me ha llamado la atención y he tratado de llevarla."

Si bien los tres profesores concordaron en que deben ser un mediador del contenido, fue posible apreciar diferencias con respecto a las otras funciones que atribuyen a su quehacer. Mientras el profesor de Historia le dio énfasis a elementos relacionados con el contenido y la clase en sí, los profesores de Física y Matemática van mucho más allá, creyendo que la función del docente no acaba en el aula, sino que implica asumir la responsabilidad de orientar a los estudiantes y promover cambios en su manera de pensar para que valoren y sientan ganas de seguir aprendiendo.

\subsection{CREENCIAS CON RESPECTO A LA PLANIFICACIÓN DEL CONTENIDO Y CONOCIMIENTOS PREVIOS}

Las principales diferencias entre los 3 docentes tienen que ver con ámbitos de la planificación y el contenido. Por un lado, se encuentra la del profesor de Historia, quien mencionó la importancia de motivar a los estudiantes al comienzo de la clase. Por otro lado, y desde el aspecto del conocimiento del contenido, el profesor de Matemática menciona 
creer que el conocimiento debe ser compartido con otros para que tenga significado. Así también, los docentes concordaron en tomar en consideración los conocimientos previos a la hora de planificar, de esta manera creen que es más fácil introducir nueva información y relacionarla de manera exitosa.

4.6. CREENCIAS CON RESPECTO AL BIENESTAR SOCIO-EMOCIONAL DE LOS ESTUDIANTES, CERCANÍA AFECTIVA Y ENTREGA DE VALORES

Con respecto al bienestar de los estudiantes, los docentes creen que un equilibrio socio-emocional promueve la concentración necesaria en clases y el aprendizaje de los contenidos que se le entregan. Directamente relacionado con esto, se pudieron encontrar las creencias basadas en la cercanía afectiva y cognitiva. Desde allí los tres docentes coincidieron en el hecho de que las relaciones cercanas con los estudiantes favorecen el buen desarrollo de la clase. Esto en el caso del profesor de Física, se manifestó de manera explícita en su práctica pedagógica, en él se pudo observar de manera directa sus creencias, ya que el trato con ellos es cercana y logra incluir elementos como el futbol, la música, las teleseries e incluso algunos chistes como herramientas dentro del aula, para lograr facilitar los procesos de aprendizaje y así mismo las relaciones entre ellos. Sin embargo, cree, en conjunto con el docente de Matemática, que deben haber límites con respecto a la cercanía que se tiene con los estudiantes, para que no se convierta en demasiado informal.

Los docentes manifiestan creer que una apropiada cercanía beneficia la entrega de contenidos y logro de resultados eficaces. Esto sucede principalmente porque la cercanía favorece que el docente se interiorice más a fondo en las cualidades, gustos e intereses de los estudiantes. Al mismo tiempo, fomenta que los docentes recepcionen de mejor manera las sugerencias o críticas que puedan reconocer los estudiantes frente a su labor profesional. De esta manera, la escucha activa que tiene el docente del estudiante puede servir como insumo a la reflexión sobre la reflexión en la práctica del profesor (Schön, 1998).

Al privilegiar la cercanía y la escucha, los docentes creen que los estudiantes se logran sentir más apoyados y acompañados tanto en los procesos de enseñanza-aprendizaje como en el ámbito personal. Los tres creen que la institución educativa debe ser un espacio de formación en base a contenidos, pero que también debe ser un lugar donde se privilegie la entrega de valores. Al analizar esta creencia en relación a otras presentadas en la investigación, se pudo dilucidar la no azarosa intención de hacer clases en un colegio católico, generando de manera implícita la búsqueda de los docentes por involucrarse en espacios en donde se sientan cómodos y rodeados de personas que basen su labor en los mismos cimientos.

\subsection{CREENCIAS EN RELACIÓN A LA ADAPTACIÓN DE METODOLOGÍAS Y LAS ESTRATEGIAS PARA PROMOVER LA MOTIVACIÓN Y PARTICIPACIÓN EN LOS ESTUDIANTES}

Uno de los trabajos pioneros en el tema de creencias docentes, es el de Wehling y Charters (Cit. en Porlán, 1995: 8), en el que se identificaron una serie de dimensiones. Una de ellas fue la adaptación a los alumnos. Según los autores, esta dimensión representa la creencia de que la enseñanza se debe organizar en tomo a los intereses y necesidades de los alumnos como forma de contribuir a su desarrollo afectivo y social. Los profesores creen que esto genera mayor motivación por parte de los estudiantes, por consiguiente 
mayor participación, mejores aprendizajes y así mismo la confianza de que de vez en cuando se pueda bromear sin faltarle el respeto nadie, sino más bien como herramienta de trabajo para el propio docente y contribuyendo al buen clima de aula. Relacionado con lo anterior y en lo referente a estrategias motivacionales y participativas, los tres docentes concordaron en que el preguntar directivamente resulta efectivo para fomentar la participación entre sus estudiantes.

Tanto en el docente de Historia como en el de Matemática apareció la creencia de reforzar los aspectos positivos y la resolución de problemas, para que de esta manera el estudiante se sienta más seguro de sus capacidades y se atreva a participar. Por otro lado, los tres docentes concordaron en que es necesario adaptar las metodologías y estrategias a los estudiantes, según sus características, necesidades y estilos de aprendizaje para contribuir a su aprendizaje. Si bien el docente de Matemática cree que es necesario adaptar las metodologías y estrategias a sus estudiantes, cree que al ser un profesor principiante no puede cumplir esto del todo, por ende, considera que muchas veces los estudiantes son los que deben adaptarse a él, ya que aún le faltan las herramientas, pero demuestra interés en hacerlo.

\subsection{CREENCIAS CON RESPECTO MANEJO DISCIPLINAR EN EL AULA Y APRECIACIÓN DE LOS ESTUDIANTES}

Con respecto al manejo disciplinar los docentes tienen creencias bastante homogéneas. Roehrig et al. (2007) menciona que los profesores ejemplares no debiesen realizar prácticas que repercutan de manera negativa en la motivación y el proceso de aprendizaje de los estudiantes. Se puede apreciar que los tres docentes creen que hay ciertos métodos que se deben utilizar para el manejo de conducta y concuerdan en algunos, como el llamado de atención, tiempo fuera, cambio de puesto, conversar con el estudiante y no anotar en el libro. A raíz de los problemas conductuales y disciplinarios que puedan presentar los estudiantes es que tanto para el docente de Física, como para el docente de Matemática, es necesario tener claro que al momento de enseñar, se deben separar las apreciaciones personales de las académicas que se tengan sobre los estudiantes. En el caso del docente de Física, considera que un impasse en el ámbito académico con sus estudiantes no debe ser interpretado como algo personal.

\subsection{CREENCIAS SOBRE EL APRENDIZAJE SIGNIFICATIVO Y CREATIVIDAD}

Es posible apreciar diferencias entre los docentes en lo que respecta al aprendizaje significativo. Para el docente de Física, un aprendizaje significativo se da cuando los estudiantes aprenden por medio de la experiencia. En cambio, para el profesor de Historia el aprendizaje significativo implica un proceso metacognitivo, donde el estudiante se vuelve consciente de que posee ciertos conocimientos y le otorga un valor significativo a éstos.

(Docente B) "un aprendizaje significativo se logra cuando a los estudiantes tú le haces click en la cabeza. ¿Y cómo puedes hacerle click a un estudiante en la cabeza? Tú puedes lograr eso, cuando tú le hablas de temas que él conoce, pero no se había dado una autoconsciencia de que lo sabía"

Por otro lado, el profesor de Matemática cree que el aprendizaje significativo se da por medio de la construcción del conocimiento junto con el trabajo constante de los 
estudiantes. Elementos que según el docente es difícil de fomentar. Los docentes de Historia y Matemática coincidieron en que los estudiantes deben cumplir un rol activo a la hora de adquirir nuevos aprendizajes, para que de esta manera, las estrategias vertidas en la clase logren ser realmente significativas. Un importante elemento que también mencionaron estos dos profesores, es el de la creatividad, a pesar de que lo hacen desde enfoques diferentes. El profesor de Historia cree que la creatividad es fundamental para que los estudiantes resignifiquen los contenidos, permitiendo así una mejor asimilación de éstos. Por otro lado, para el docente de Matemática resulta fundamental que el profesor sea creativo, con el fin de que ello le permita ser capaz de presentar un mismo contenido de diferentes formas, las veces que sea necesario hasta lograr el aprendizaje en sus estudiantes.

\subsection{CREENCIAS SOBRE EL USO DE HERRAMIENTAS}

PRÁCTICAS Y EJEMPLIFICACIÓN DE LOS CONTENIDOS

Las creencias con respecto a las herramientas didácticas son de gran importancia al momento de llevar el conocimiento que posee el docente a las prácticas pedagógicas, o sea del conocimiento didáctico del contenido (Shulman, 2005). Para los tres docentes las creencias sobre la utilización de métodos de carácter más práctico se basan en actividades que están cargadas de elementos provenientes de las realidades de los estudiantes. Esto permite hacer concretos contenidos que son más abstractos y difíciles, como por ejemplo las fórmulas (Física) y poder manipular y resignificar la información (Historia). El docente de Matemática manifestó creer que su uso es imperioso cuando las metodologías más tradicionales no logran que el estudiante comprenda. En general, esto se reflejó en las prácticas dentro del aula de los docentes al ver que más que cases expositivas, las temáticas fueron abordadas con diversas actividades que implicaban ejemplos relacionados con la vida cotidiana de los estudiantes y aplicación de la materia en situaciones concretas: mostrar un resorte "real" para explicar lo que es y cómo funciona, generando concordancia entre su discurso y su quehacer profesional. De manera similar ocurrió con la utilización de ejemplos, los tres docentes creen que la aplicación de esta herramienta debe estar basada en la cotidianeidad de los estudiantes, lo cual fomenta la motivación por los contenidos y se adquieren los aprendizajes de manera más significativa, tomando en consideración que los contenidos únicamente vistos desde la teoría resultan más difíciles de asimilar.

\subsection{CREENCIAS CON RESPECTO A LA DIVERSIDAD EN EL ESTUDIANTE Y ESTILOS DE APRENDIZAJE}

Al hablar sobre los estilos de aprendizaje, los tres docentes concordaron en que creen y reconocen la existencia de diferentes estilos de aprendizaje y que éstos deben ser tomados en consideración al momento de escoger las metodologías a utilizar con los estudiantes.

(Docente B) "Obviamente las diferencias individuales son claves, no todas las personas aprenden de la misma manera"

Con esto se vincula la diversidad de capacidades que existen entre los jóvenes y las expectativas que tienen los docentes con respecto a ellos. Los docentes de Física e Historia mencionaron creer tener altas expectativas con respecto a sus estudiantes, lo cual 
se relaciona directamente con las exigencias que imponen. Como creen que son capaces de muchas cosas las exigencias son cada vez más altas. Las derrotas con respecto a las capacidades creen poder atribuirlas a la flojera (Física) o a las dificultades que algunos puedan tener para asimilar los contenidos, que no tengan que ver ni con motivación o participación (Matemática). Como manera de revertir la flojera descrita por el docente de Física, él procura hacer participar a quienes no quieran hacerlo por cuenta propia, considerando que para este profesor la participación no implica solamente dar su opinión levantando la mano, sino que tiene que ver con cualquier actividad que se desarrolle en el contexto de la asignatura tanto fuera como dentro del aula.

\subsection{CREENCIAS CON RESPECTO A LAS CUALIDADES DE ESTUDIANTE}

Con respecto a la autonomía de estudiante, entendida como la concepción del profesor sobre el nivel de control que es conveniente mantener en los procesos de enseñanzaaprendizaje (Cit. en Porlán, 1995: 8), los tres docentes creen que los estudiantes deben ser parte activa de los procesos que se dan en su educación, el docente debiese ser un proveedor de herramientas para que los jóvenes puedan investigar, descubrir, reflexionar, comprender y aprender, sin convertirse en un mero reproductor del conocimiento. El docente de Matemática cree además que no se debe reforzar de manera inmediata diciendo al estudiante si se encuentra en lo correcto o no. El profesor de Física, por su parte, cree que como están inmersos en un sistema que no propicia la autonomía, esta debe ser integrada de manera paulatina, para que al final entiendan que los fines no son las notas, sino que va mucho más allá de aquello.

Por último, el docente de Historia cree que es importante dejar que la autonomía esté presente en situaciones como la elección de temáticas en trabajos y exposiciones, de acuerdo a sus intereses personales, creando de esta manera un espacio creativo para los estudiantes, donde el aprendizaje se dé en la medida que utilicen sus habilidades y las desarrollen como más les acomode.

El docente de Física cree que las motivaciones que mueven al estudiante dentro del ámbito educativo son las notas, que sin estas no se interesan por las actividades. Lo cual perjudica directamente la manera en que él ve el proceso de enseñanza aprendizaje.

Asimismo pasa también con la participación de los estudiantes: los tres profesores, de Física, Historia y Matemática, creen que la participación es relevante en el logro de mejores aprendizajes, pero que la responsabilidad de que los estudiantes participen pasa por ellos mismos, que como docentes deben ser quienes los motiven para que los estudiantes se atrevan a ser más activos durante las clases. Esto lo consideran un desafío que es importante alcanzar. Tanto para el docente de Matemática como el de Física existiría una relación entre la etapa de desarrollo del estudiante y su comportamiento (participación, motivación, etc.). Esto explicaría según los docentes, la falta de motivación y ganas de los estudiantes más grandes por estar en clase. Por esta razón, tal como lo señala el profesor de Física, el docente debe cambiar de rol de acuerdo a la etapa de desarrollo en que se encuentren sus estudiantes. Para estos mismos docentes (Matemática y Física), el éxito se alcanza con esfuerzo y dedicación pasando a segundo plano las habilidades que la persona pueda tener.

En definitiva si bien los docentes reconocieron que los estudiantes poseen distintas capacidades y habilidades sería el esfuerzo que éstos ponen en los estudios lo que realmente garantiza el éxito. 
Las principales creencias encontradas por medio del análisis de los datos tuvieron estrecha relación con las prácticas observadas dentro del aula. La existencia de algunas inconsistencias fueron reconocidas por ellos mismos en su discurso, atribuyéndolas a la falta de experticia. Esto da a conocer que los docentes poseen procesos activos de reflexión sobre sus prácticas, lo cual posibilita la superación de dificultades y el autoaprendizaje. Si bien en este contexto se encontraron algunas creencias negativas frente a la educación, ninguno fue dirigido a su desempeño o hacia los estudiantes, sino que más bien al contexto político y económico que enmarca a la educación en Chile.

$\mathrm{Al}$ ser profesores noveles, se ha encontrado un amplio interés por seguir perfeccionando su labor, aspirando a convertirse en excelentes docentes en el futuro. En este proceso ha influido la presencia de personas significativas dentro de su formación, las cuales han gatillado diferencias considerables entre lo que consideran un buen y un mal profesor. Cabe mencionar que aunque quizás ellos no lo reconozcan, poseen diversas características que los sitúan dentro de lo que se llama profesores ejemplares, a pesar de su poca experiencia, lo cual es bastante meritorio si se mira objetivamente, ya que hay habilidades que no se esperan encontrar en los profesores después de una considerable trayectoria.

Tanto el discurso como las prácticas fueron concordantes a la hora de referirse a la apreciación que se tiene de los estudiantes. En general esta influencia por el carácter valórico de la institución donde trabajan, se basa en fomentar el respeto y la integridad de las personas que son parte de la comunidad educativa. De esta manera, se procura ver a los estudiantes no solamente desde lo académico, sino que también desde lo personal. Las expectativas son altas, pero se trabaja en base al pensamiento que los estudiantes son los protagonistas de los procesos de enseñanza aprendizaje.

\section{CONCLUSIÓN}

En esta investigación se pudo dar cuenta de las principales y más relevantes creencias sobre los procesos de enseñanza aprendizaje encontrándose creencias en torno a la identidad profesional de los docentes, la enseñanza y las características de los estudiantes.

Por otro lado, se evidenciaron creencias relacionadas directamente con características propias de profesores ejemplares, tales como interés por la participación de los estudiantes, utilización de distintas estrategias y metodologías de enseñanza, considerando las opiniones, demandas, necesidades individuales de los estudiantes, cercanía afectiva con sus estudiantes, gusto por la enseñanza, expectativas positivas de los estudiantes, entre otras. Lo cual resulta importante de destacar, ya que va en concordancia con los hallazgos de diferentes autores como Porlán, (1995), López (2010), Harper (2006) Brophy y Good (Cit. en Roehrig et al. 2007: 1-19) y Cruz (2008).

La concientización de las creencias es el primer paso para que éstas sean reformuladas y mejoradas. Al conocer las similitudes y diferencias que poseen profesores ejemplares con respecto a sus creencias sobre la educación y la enseñanza, al ser comparadas con sus prácticas, fue posible discriminar entre aquellas que están dando resultados positivos y aquellas que podrían ser mejoradas en pos de entregar una educación de mayor calidad. Esta información podría servir de base para generar nuevas propuestas de formación del profesorado, difundiendo de esta manera buenas prácticas dentro del gremio. 
Cabe mencionar que los tres docentes convergieron en poseer un nivel de reflexión técnica y metodológica acerca de la pedagogía y además un ideario ético, desde el cual consideran las implicancias valóricas de su rol y de los procesos de enseñanza-aprendizaje. Esto en cierta medida ha influido en el hecho que desean formar estudiantes como personas íntegras, tratando de promover la idea que la educación no es simplemente una entrega de contenidos, sino que también puede ser un medio por el cual inculcarles ciertos valores.

Los participantes también coincidieron en poseer una proporción mayor de creencias positivas sobre los procesos educativos y sus actores, en contraposición de aquellas que tienen un carácter más negativo. De hecho, en el presente estudio fueron escasas las creencias de carácter negativo detectadas y constituyeron más bien excepciones a un acervo centrado en características positivas de la enseñanza, el educando y el pedagogo.

Por otro lado, si bien se habla de profesores noveles, se evidenció que tanto en el discurso como en la práctica, dos años más de experiencia pueden determinar una diferencia significativa entre ellos. Esto se da en ámbitos como la seguridad que presentó el docente al enfrentarse a un curso, como también en lo que respecta a la concordancia existente entre el discurso y la práctica. Esto está íntimamente relacionado con las creencias y prácticas que tienen que ver con el manejo disciplinar y el control de grupo. Sin embargo, lo anterior no lo atribuyeron a carencias en la formación o al contexto psicoeducativo en el que se desenvuelven; sino a la escasa práctica docente. Una última creencia se relaciona con la insatisfacción que le produce al docente, la falta de motivación de sus estudiantes o la motivación extrínseca que generalmente estos poseen hacia en aprendizaje.

En base a lo señalado anteriormente, la investigación se encontró frente a una dicotomía experto-novel versus la categoría de profesor "ejemplar". A partir de esto se pudo obtener inferencias que van más allá de los casos particulares estudiados. Como ya es sabido, los profesores participantes de esta investigación son considerados noveles, por sus años de experiencia. Sin embargo, se ha evidenciado en ellos características de docentes ejemplares, tales como la adaptación de metodologías y estrategias; el llevar a cabo la labor pedagógica de manera autorreflexiva; poseer la capacidad de innovar y adaptar el currículo; entre otras. Este hallazgo resulta interesante de ser abordado, ya que usualmente la literatura asocia dichas características a profesores expertos, los cuales por medio de la experiencia han logrado tener mayor claridad con respecto a sus prácticas y a las herramientas más idóneas para obtener mejores aprendizajes en sus estudiantes. De esta manera, se puede decir que la calidad de ejemplar no es sólo atribuible a profesores expertos, sino que también a docentes que recién han comenzado su trayectoria profesional.

Además, los hallazgos nos indicaron que es muy probable que las creencias que los pedagogos expresan, directa o indirectamente, guíen su toma de decisiones y no son simplemente un discurso desacoplado de sus prácticas. Hay dos razones para concluir esto. En primer lugar, se observó un grado importante de coherencia entre las creencias expresadas por los profesionales de la educación y las prácticas pedagógicas que fueron observadas en la sala de clases. En segundo lugar, el dinamismo que las creencias tuvieron, según los relatos manifestados por los participantes de este estudio, revela que los saberes de los pedagogos no son simplemente líneas aprendidas de memoria en la universidad, sino que se adaptan, se cuestionan y sufren cambios a partir de la práctica de la pedagogía. Así, es muy plausible que se produzca una retroalimentación constante entre saber y práctica, que justifica tanto las reestructuraciones de las creencias y reflexiones sobre el tema educativo como las decisiones tomadas en el aula. 
En otras palabras, si se pretende mejorar los procesos de enseñanza-aprendizaje, resulta fundamental que los docentes reflexionen sobre las creencias que se encuentran a la base de sus prácticas para que de este modo, se puedan llevar a cabo acciones de reconstrucción a nivel de aula con el fin de mejorar por consiguiente su docencia. Como menciona Schön (1998) cuando el docente logra una metareflexión sobre su práctica, se produce un análisis tanto de las situaciones, como también de los procedimientos, la toma de decisiones, las teorías implícitas, la manera de acercar el contenido a los estudiantes, etc. Así el estudio y descripción de las creencias docentes pueden servir para proponer cambios en la formación inicial y permanente del profesorado. Resultaría conveniente y sumamente beneficioso continuar realizando estudios dentro de esta misma línea y con docentes percibidos como ejemplares, ya que de esta forma no sólo será posible corroborar resultados de investigaciones anteriores, sino también abre la posibilidad de encontrar nuevas creencias y elementos positivos para fomentarlos en el profesorado, ya sea en forma de capacitaciones o durante el transcurso de la carrera de pedagogía.

\section{REFERENCIAS BIBLIOGRÁFICAS}

Arzaluz, S. (2005). La utilización del estudio de caso en el análisis local. Región y Sociedad, vol. XVII, $n$. 32, 107 - 144. Web: http://lanic.utexas.edu/project/etext/colson/32/4araluz.pdf (05/2011).

Castro, J. (2007). Experiencias Didácticas para el Mejoramiento de la Práctica Pedagógica del Profesor de Artes Plásticas. Educación, vol. 31, n. 001, 109-121. Web: http://www.redalyc. org/redalyc/pdf/440/44031107.pdf (06/2011).

Cruz, I. (2008). Creencias pedagógicas de profesores: el caso de la licenciatura en nutrición y ciencia de los alimentos en México. Revista Qurriculum, vol. 21, 137-156. Web: http://webpages.ull. es/users/revistaq/ANTERIORES/numero21/cruz.pdf (10/2011).

Day, C. (2006). Por qué es esencial la pasión. En C. Day (Ed.), Pasión por enseñar: la identidad personal y profesional del docente y sus valores (pp. 27-39). Madrid: Narcea.

De Vincenzi, A. (2009). Concepciones de enseñanza y su relación con las prácticas docentes: un estudio con profesores universitarios. Educación y Educadores, vol. 12, n. 2, 87-101. Web: http://www.redalyc.org/articulo.oa?id=83412219006 (05/2011).

Denzin, N. y Lincoln, Y. (1994) Introduction: entering the field of Qualitative Research. En N. Denzin, E. y. Lincoln (Ed.), Handbook of Qualitative Research (pp. 1-34). California: Sage Publications.

Denzin, N. y Lincoln, Y. (2008). The landscape of Qualitative Research (3 ${ }^{\mathrm{a}}$ edición). London: Sage Publications.

Díaz, F. y Núñez, P. (2008). Formación y evaluación de profesores novatos: Problemática y retos. México: Universidad Autónoma Metropolitana - Xochimilco. Web: http://148.206.107.15/ biblioteca_digital/articulos/3-303-4716cyg.pdf (12/2011).

Díaz, L., Martínez, I., Roa, G. y Sanhueza, J. (2010). Los docentes en la sociedad actual: sus creencias y cogniciones pedagógicas respecto al proceso didáctico. Polis, vol. 9, n. 25, 421436. Web: http://www.scielo.cl/pdf/polis/v9n25/art25.pdf (05/2011).

Fierro, C.; Fortour, B.; Rosas, L. (1999). Transformando la práctica docente. Una propuesta basada en la investigación-acción. México: Paidós.

Gutiérrez, J. (2008). ¿Cómo reconocemos un buen maestro? Revista mexicana de investigación educativa, vol. 13, n. 039, 1299-1303. Web: http://www.redalyc.org/articulo.oa?id=14003914 $(08 / 2011)$.

Guzmán, C.; Rodríguez, V.; Imbernon, F.; De La Cerda, C. y Carrasco, C. (2011). Prácticas pedagógicas virtuosas: los casos de docentes de Liceos Prioritarios de la región de Valparaíso (Chile). 
En MINEDUC, Evidencias para políticas públicas en educación. Selección de investigaciones tercer concurso FONIDE (pp. 187-226). Santiago de Chile: MINEDUC

Harper, L. (2006). A Teacher's Re-definition of Elementary Level Teaching. Institute for Learning Centered Education. Web: http://www.jpacte.org/uploads/9/0/0/6/9006355/2006-1-harper.pdf (09/2011).

Imbernón, F. (1994). La formación permanente y el desarrollo profesional del profesorado. En F. Imbernon (Ed.), La formación y el desarrollo profesional del profesorado: Hacia una nueva cultura profesional (pp. 58-59). Barcelona: Graó.

Latorre, M. (2004) Aportes para el análisis de las racionalidades presentes en las prácticas pedagógicas. Revista Estudios Pedagógicos, n. 30, 75-91.

López, S. (2010). Las complejidades emergentes en las historias de vida de los "buenos profesores". Polis, vol. 9, n. 25, 255-267.

Marcelo, C. (2009). Profesores principiantes y programas de inducción a la práctica docente. España: Universidad de Salamanca. Web: http://gredos.usal.es/jspui/bitstream/10366/69317/1/ Profesores_principiantes_y_programas_de_.pdf (11/2011).

Ochoa, S., Domínguez, A. (2007). Buenos Maestros vs. Malos Maestros. Psicología Iberoamericana, vol. $15, n .2,11-16$

Oliver, C (2009). El valor formativo y las ataduras de las creencias en la formación del profesorado. Aquello que no se ve, pero se percibe en el aula. Reifop, vol. 12, n. 1, 63-75.

Osses, S., Sanchez, I. e Ibañez, F. (2006). Hacia la generación de teoría a través del proceso analítico. Estudios Pedagógicos, vol. 32, n. 1, 119-133.

Patton, M. (1990). Qualitative interviewing. En M. Patton (Ed.), Qualitative evaluation and research methods. Newbury Park, CA: Sage Publications.

Porlán, R (1995). Fundamentos Conceptuales y Didácticos. Las creencias pedagógicas y científicas de los profesores. Enseñanza de las ciencias de la tierra, vol. 3, n. 1, 7-13.

Roehrig, A., Bohn, C., Turner, J. \& Pressley, M. (2007). Mentoring beginning primary teachers for exemplary teaching practices. Teaching and Teacher Education, vol. 24, n. 3, 1-19.

Segovia, J. (2003). Dimensiones y escenarios del buen aprendizaje para todos. Revista Electrónica Iberoamericana sobre Calidad, Eficacia y Cambio en Educación, vol. 1, n. 2, 1-21.

Schön, D. (1998). El profesional reflexivo. Cómo piensan los profesionales cuando actúan. Barcelona: Paidós.

Shulman, L. (2005). Conocimiento y enseñanza: fundamentos de la nueva reforma. Revista de Currículum y Formación del Profesorado, vol. 9, n. 2, 1-30.

Vasilachis, I. (2006). Estrategias de investigación cualitativa. Barcelona: Gedisa. 
